Rosales-Contreras, G.I., Laguarda-Figueras, A. \& Solís-

Marín, F.A. (2021). Morfología y microestructura interna de la estrella quebradiza Ophiocomella alexandri (Echinodermata: Ophiocomidae). Revista de Biología Tropical, 69(S1), 358-374. DOI 10.15517/rbt.v69iSuppl.1.46367

DOI 10.15517/rbt.v69iSuppl.1.46367

\title{
Morfología y microestructura interna de la estrella quebradiza Ophiocomella alexandri (Echinodermata: Ophiocomidae)
}

\author{
Gloria Itzel Rosales-Contreras ${ }^{1 *}$ \\ Alfredo Laguarda-Figueras ${ }^{2}$ \\ Francisco A. Solís-Marín ${ }^{2}$
}

1. Posgrado en Ciencias del Mar y Limnología, Instituto de Ciencias del Mar y Limnología, Universidad Nacional Autónoma de México, Av. Universidad, Circuito Exterior s/n, Alcaldía Coyoacán, Ciudad de México, México; roscongloitz@hotmail.com

2. Laboratorio de Sistemática y Ecología de Equinodermos, Instituto de Ciencias del Mar y Limnología, Universidad Nacional Autónoma de México, Av. Universidad, Circuito Exterior s/n, Alcaldía Coyoacán,, Ciudad de México, México; laguarda@cmarl.unam.mx, fasolis@cmarl.unam.mx

$$
\text { Recibido 11-VII-2020. Corregido 25-XI-2020. Aceptado 20-XII-2020. }
$$

\section{ABSTRACT \\ Morphology and internal microstructure of the brittle star Ophiocomella alexandri \\ (Echinodermata: Ophiocomidae)}

\begin{abstract}
Introduction: Ophiocomella alexandri is a conspicuous species in the Eastern Tropical Pacific. The diagnosis is based on adult specimens and there are not documented variations. The inclusion of internal microstructures to the descriptions will allow intraspecific and interspecific comparisons in taxa with taxonomic problems. Objective: To describe the external morphology and internal microstructures of $O$. alexandri, including variation. Methods: We reviewed $O$. alexandri type material and general material deposited in three scientific collections. We examined the internal microstructures of the arm and jaw with a scanning electron microscope. Results: The external and internal morphology were described showing the morphological variations according to size. Conclusions: The variations in the internal morphology of $O$. alexandri show that this type of analysis is necessary, and even more important than the external morphology. It is recommended to carry out more taxonomic work that includes other species of the genus to clarify its taxonomic status.
\end{abstract}

Key words: arm; jaw; characters; variations; scanning electron microscopy.

Ophiocomella alexandri (Lyman, 1860) es una especie conspicua del Pacífico Oriental Tropical (POT); a pesar de ello, sus variaciones morfológicas no han sido documentadas y su identificación taxonómica no ha sido estricta, ya que no se ha prestado atención en los caracteres diagnósticos como estructuras del brazo y mandíbula, los cuales son variables en forma y número. Comúnmente suele reconocerse a $O$. alexandri por tener granulación espinosa en el disco, coloración marrón-beige y bandas marrones en los brazos diferenciándola de quien era su único congénere en el POT Ophiocoma aethiops Lütken, 1859.

Lyman en 1860 describe por primera vez a $O$. alexandri utilizando un ejemplar adulto de $17 \mathrm{~mm}$ de diámetro de disco (DD) por lo que la diagnosis de la especie en muchas ocasiones no es correspondiente con ejemplares de menor tamaño. Por otro lado, existen alrededor de 30 
trabajos que reportan a O. alexandri; sin embargo, se han limitado al registro para un área geográfica particular (e.g. Clark, 1913; Birkeland, Meyer, Stanes \& Buford, 1975, Solís-Marín, Reyes-Bonilla, Herrero-Pérezrul, Arizpe-Covarrubias \& Laguarda-Figueras, 1997), al uso de la diagnosis, o bien, no detallan los caracteres diagnósticos (e.g. Clark, 1921; Boone, 1926; Nielsen, 1932, Cortés-Fernández, 1999). En el caso de los trabajos donde se han realizado descripciones más detalladas, la forma de las estructuras (e. g. placas dorsales y ventrales de los brazos) y el número de las papilas asociadas a la mandíbula mencionadas por cada autor han sido variables y no se han mencionado tales variaciones, a excepción de la coloración. (e.g. Caso, 1951; Granja-Fernández, Herrero-Pérezrul, López-Pérez, Hernández, Rodríguez-Zaragoza, Jones \& Pineda-López, 2014; Conejeros-Vargas, 2015).

La existencia de las colecciones científicas, sumado a la gran abundancia de esta especie, ha provisto material el cual ha permitido observar su variación morfológica y documentarla. Aunado a esto, en los últimos años, el uso de microestructuras internas del brazo y la mandíbula se ha convertido en una herramienta importante e indispensable para identificar a las especies de la clase Ophiuroidea (Martynov, 2010; Hendler, 2018). La inclusión de estos caracteres internos en las descripciones permite hacer comparaciones intra e interespecíficas en grupos con problemas taxonómicos. Recientemente la familia Ophiocomidae tuvo cambios taxonómicos importantes, entre los cuales, $O$. alexandri fue transferida del género Ophiocoma al género Ophiocomella (O'Hara et al., 2019), en consecuencia, resulta de alta importancia caracterizarla adecuadamente.

Por lo anterior, el presente trabajo tiene como objetivo describir la morfología externa y microestructuras internas de $O$. alexandri, incluyendo sus variaciones morfológicas.

\section{MATERIALES Y MÉTODOS}

Se revisó el material de la especie $O$. alexandri (2335 ejemplares) depositado en la
Colección Nacional de Equinodermos "Dra. Ma. Elena Caso Muñoz", Instituto de Ciencias del Mar y Limnología, Universidad Nacional Autónoma de México, México (ICMLUNAM) y el United States Natural History Museum, Smithsonian Institution, Washington, D.C., E.U.A. (USNM), así como el material tipo depositado en el Museum of Comparative Zoology, Harvard University (MCZ).

Para la observación de variaciones intraespecíficas de las microestructuras internas de brazo y mandíbula se disectaron 48 ejemplares de diámetro del disco (DD) $>10 \mathrm{~mm}$ y cuatro ejemplares de DD $<6 \mathrm{~mm}$. Los lotes de los ejemplares disectados se señalan con un asterisco en el material suplementario (ver Apéndice 1). El aislamiento de las placas calcáreas se realizó utilizando una solución de hipoclorito de sodio al $50 \%(\mathrm{NaClO})$ con la cual se disolvió el tejido. Una vez que las placas se desarticularon fueron sometidas a un tren de deshidratación (agua corriente, agua destilada y alcohol al $100 \%$ ) y se prepararon para su observación en el microscópio electrónico de barrido modelo Hitachi SU1510 del Laboratorio de Microscopía y Fotografía de la Biodiversidad I, del Instituto de Biología de la Universidad Nacional Autónoma de México, UNAM. Para que los resultados fueran comparables, se disectó el quinto segmento del brazo (contando a partir del primer segmento dorsal) y un quinto del disco localizado a la derecha de la madreporita en todos los ejemplares.

Se describió la morfología externa e interna de los ejemplares. Las estructuras y sus acrónimos se mencionan a continuación: primera placa ventral de los brazos $\left(1^{\circ} \mathrm{PV}\right)$, escudo oral (EO), escudo adoral (EAD), placa oral (PO), escudo radial (ER), placa genital abradial (PGab), placa genital adradial (PGad) y aleta (A). En la descripción de la morfología interna de las microestructuras del brazo se empleó la terminología propuesta por LeClair (1996): proceso aboral (PRA), proceso oral (PRO), proceso medio (PRM), faceta articulada (FA), silla media (SM), cuenca media (CM), canal radial $(\mathrm{CR})$, área muscular oral $(\mathrm{MuO})$, área muscular aboral (MuA) surco aboral (SA), articulación 
de la espina (AES), cóndilo (CO), perforación muscular $(\mathrm{Mu})$ y perforación nerviosa $(\mathrm{N})$.

En la descripción de la morfología interna de la mandíbula y las papilas orales asociadas se empleó la terminología propuesta por Hendler (2018), las cuatro papilas orales fueron nombradas desde la más externa hasta la más interna de la siguiente forma: osículo de Lyman (OLy), espina del escudo adoral (Eead), segunda espina del escudo adoral $\left(2^{\circ}\right.$ Eead), papila infradental $(\mathrm{PaI})$, papila dental $(\mathrm{PaD})$ y dientes (Di). Para describir la placa oral se usaron los siguientes términos: primer compartimiento del tentáculo oral (CTO1), segundo compartimiento del tentáculo oral (CTO 2) y meseta de la espina de la placa oral (MEPO), área de articulación adradial (AAad), área muscular abradial (Mab), área muscular adradial (Mad), cóndilo $(\mathrm{CO})$, perforación nerviosa $(\mathrm{N})$, área papilar (AP) y septum (S).

\section{RESULTADOS}

\section{Taxonomía}

Filo Echinodermata Bruguière, 1791

Clase Ophiuroidea Gray, 1840

Orden Ophiacanthida O'Hara et al., 2017

Familia Ophiocomidae Ljungman, 1867

Género Ophiocomella A.H. Clark, 1939

Ophiocomella alexandri (Lyman, 1860)

(Fig. 1A-G, Fig. 2A-D, Fig. 3A-O, Fig. 4A-O, Fig. 5A-N)

Ophiocoma alexandri Lyman, 1860: 256257; Lyman, 1865: 71, 74-75; Lyman, 1882: 170-171, 311, 325; Verrill, 1867: 259; Ives, 1889: 177; Koehler, 1907: 325; Koehler, 1914: 116; H.L. Clark, 1913: 217; H.L. Clark, 1915: 291; H.L. Clark, 1917: 440; H.L. Clark, 1921: 131; H.L. Clark, 1923: 157; H.L. Clark, 1940: 34; Campbell, 1921: 48; Boone, 1926: 7; Nielsen, 1932: 248; Ziesenhenne, 1937: 227; Steinbeck \& Ricketts, 1941: 387; Caso, 1951: 219-220, 235-241; Caso, 1961: 143-149; Caso, 1979: 208-210; Caso, 1992: 38-42; Downey, 1969: 103; Devaney, 1970: 29; Birkeland et al., 1975: 68; Brusca, 1980: 407-408; Luke, 1982:
32; Maluf, 1988: 64; Anzo, 1994: 29; Caso, Laguarda-Figueras, Solís-Marín, Ortega-Salas, \& Durán-González, 1996: 109; Solís-Marín et al., 1997: 254; Solís-Marín, Laguarda-Figueras, Durán-González, Ahearn \& Torres-Vega, 2005: 127; Kerstitch \& Bertsch, 2007: 112; Honey-Escandón, Solís-Marín \& LaguardaFigueras, 2008: 63; Granja-Fernández, 2010: 26-27; Pineda-Enríquez, 2011: 86-87; GranjaFernández, et al., 2014: 129-132; ConejerosVargas, 2015: 115-117.

Ophiocomella alexandri.- O'Hara et al., 2019: 71-75; Granja-Fernández \& Hooker, 2020: 549-550.

Localidad tipo: Acapulco, Guerrero, México (Lyman, 1860).

Material tipo examinado: 42 ejemplares. Holotipo: MCZ OPH-1825 (uno). Paratipos: MCZ OPH-1663 (cuatro), MCZ OPH-4106 (uno), USNM 1171 (10), USNM 3011 (15), USNM 12757 (11) (Apéndice 1).

Material suplementario examinado: 2293 ejemplares, 376 lotes (Apéndice 1).

Diagnosis (modificada de Lyman, 1860): Disco y ER cubiertos por gránulos espiniformes que se extienden hasta la base de los brazos. Aproximadamente $16 \mathrm{PaD}$ muy juntas, las más ventrales se encuentran en conglomerado y el resto se distribuyen en dos o tres líneas verticales. EO redondos y ligeramente truncados proximalmente. EAD en forma de moño dispuestos a los lados del EO. Brazos con bandas transversales marrón claro. Placas dorsales de los brazos cordiformes con bordes redondeados, en algunos casos triangulares. Placas ventrales de los brazos cuadrangulares con bordes laterales cóncavos y se sobreponen ligeramente. Escamas tentaculares ovaladas, dos o tres en los tres primeros segmentos, pueden extenderse hasta el octavo segmento, posteriormente solo una. De cuatro a siete espinas de los brazos, largas, delgadas y romas.

Descripción externa: Holotipo $\mathrm{MCZ}$ OPH-1825. DD $=17 \mathrm{~mm}$. Disco redondo cubierto densamente por gránulos espiniformes alargados y separados que se extienden hasta la base de los brazos, debajo de ellos existen escamas imbricadas (Fig. 1B, 1C). Escudos 
radiales cubiertos por gránulos (Fig. 1B). Cinco brazos largos, de $120 \mathrm{~mm}$ de largo y $2.3 \mathrm{~mm}$ de ancho. Placas dorsales de los brazos proximales y medias, cordiformes con bordes redondeados; segmentos distales con placas triangulares (Fig. 1D).

Interradio con granulación espiniforme que disminuye en densidad proximalmente hacia la mandíbula y a las hendiduras genitales, dejando descubiertas las escamas. Hendiduras genitales alargadas, se extienden desde el borde del disco hasta el EO, presentan una hilera de escamas imbricadas y escasos gránulos en el margen (Fig. 1E). EO redondos, casi igual de anchos que de largos, ligeramente truncados en la parte proximal. Escudo que porta la madreporita

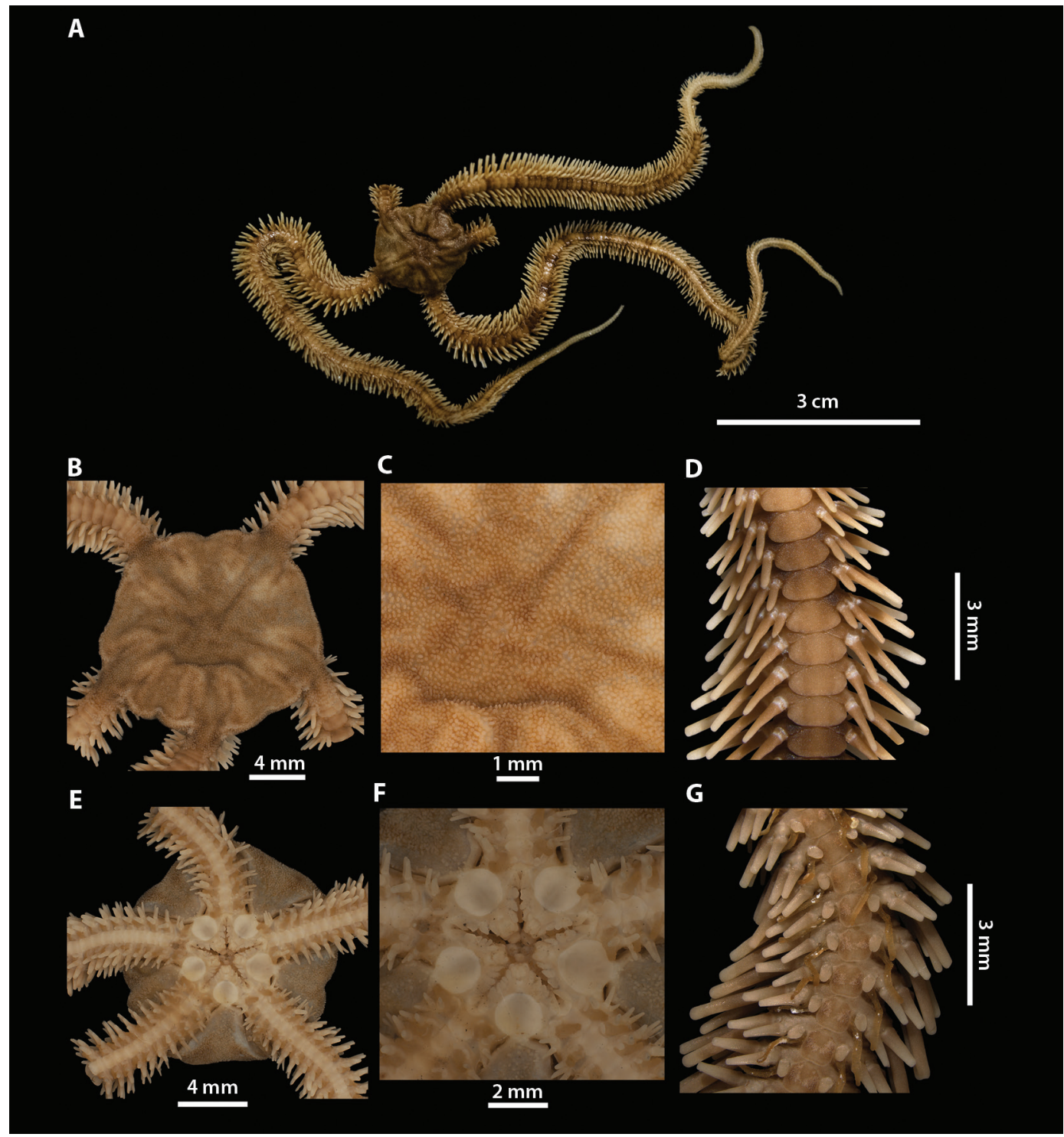

Fig. 1. Ophiocomella alexandri (Holotipo MCZ OPH-1825, DD = $17 \mathrm{~mm}$ ). A. Vista dorsal. B. Disco dorsal. C. Gránulos del disco. D. Brazo dorsal. E. Disco ventral. F. Mandíbula. G. Brazo ventral.

Fig. 1. Ophiocomella alexandri (Holotype MCZ OPH-1825, DD $=17 \mathrm{~mm}$ ). A. Dorsal view. B. Dorsal disc. C. Disc granules. D. Dorsal arm. E. Ventral disc. F. Jaw. G. Ventral arm. 
visible, de mayor tamaño, abultado, con una mancha circular más clara. EAD con forma de moño, dispuestos a los lados del EO, se extienden hasta la hendidura genital. Cuatro papilas orales a cada lado de la mandíbula y un conglomerado de papilas dentales: OLy con forma de aleta y dispuesto entre la $1^{\circ} \mathrm{PV}, \mathrm{EAD}$ y placa oral PO; Eead lanceolada y ancha; $2^{\circ}$ Eead lanceolada, ligeramente más larga que la Eead y delgada; PaI alargada y roma (Fig. 1F, Fig. 2A, Fig. 2B); nueve PaD redondeadas, muy juntas; de las nueve, cuatro papilas apicales se encuentran en conglomerado y son ligeramente más pequeñas, el resto se distribuyen en dos o tres líneas verticales. Tres o cuatro Di robustos, cuadrangulares y lisos, el más ventral ligeramente cónico (Fig. 1F, Fig. 2B).

$1^{\circ} \mathrm{PV}$ pequeña y en contacto con el OLy. Placas ventrales cuadrangulares con bordes laterales cóncavos (Fig. 1G) y un ápice proximal generalmente cubierto por la placa anterior. Placas laterales prominentes, en forma de "C" en contacto con las placas dorsales y ventrales de los brazos. Escamas tentaculares ovaladas, dos en los primeros tres segmentos y posteriormente solo una. De cuatro a siete espinas de

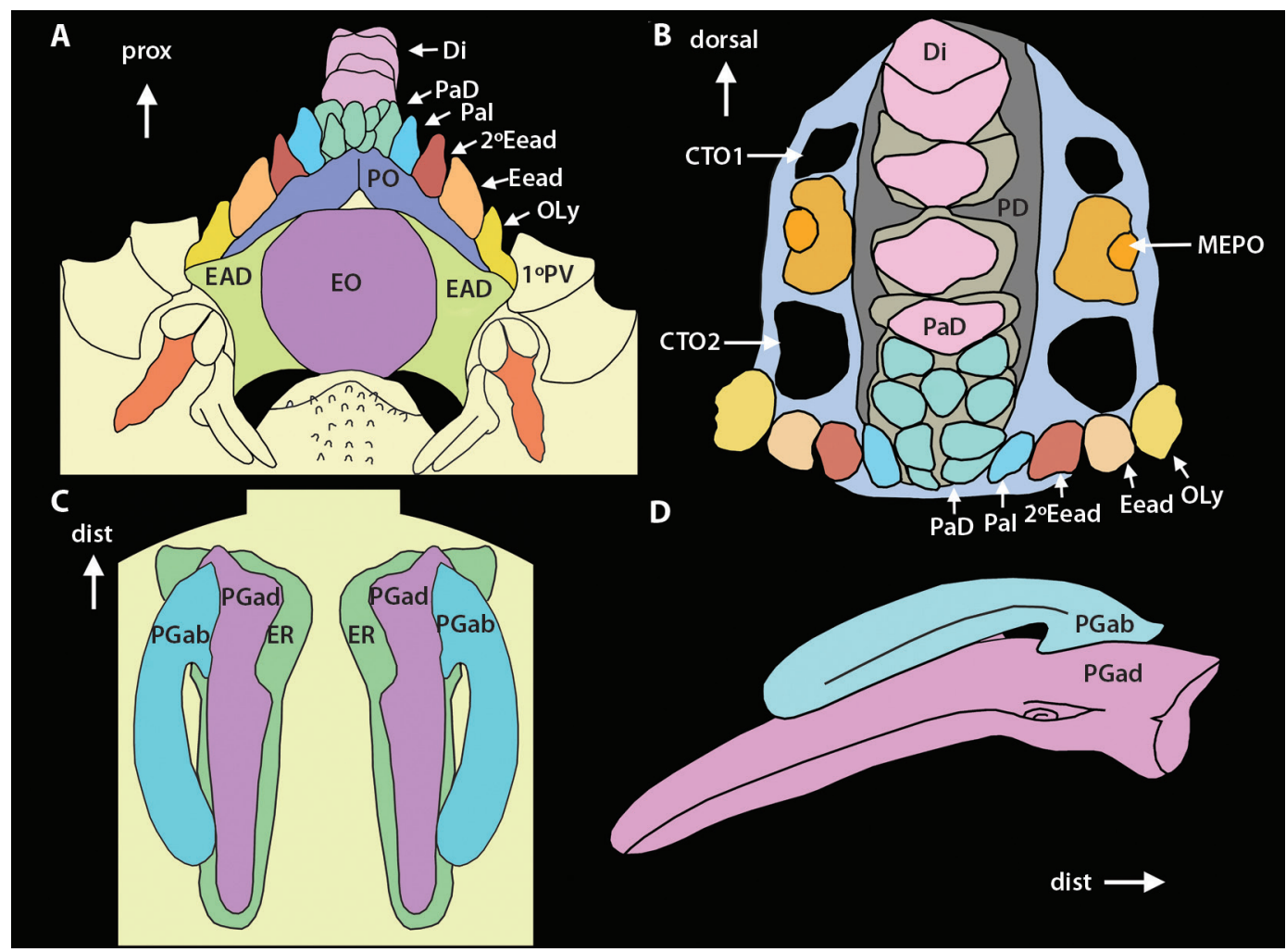

Fig. 2. Ophiocomella alexandri. Esquemas de la estructura externa e interna. A. Mandíbula, vista ventral. B. Mandíbula, vista transversal. C. Placas genitales articulando con el escudo radial. D. Placas genitales. CTO1. Primer compartimiento del tentáculo oral. CTO2. Segundo compartimiento del tentáculo oral. Di. Dientes. EO. Escudo oral. EAD. Escudo adoral. Eead. Espina del escudo adoral. $2^{\circ}$ Eead. Segunda espina del escudo adoral. MEPO. Meseta de la espina de la placa oral. OLy. Osículo de Lyman. PaD. Papilas dentales. PaI. Papila infradental. PD. Placa dental. PGab. Placa genital abradial. PGad. Placa genital adradial. PO. Placa oral. $1^{\circ} \mathrm{PV}$. Primera placa ventral.

Fig. 2. Ophiocomella alexandri. Diagrams of the internal structure. A. Jaw, ventral view. B. Jaw, cross-sectional view. C. Genital plates articulating with the radial shield. D. Genital plates. CTO1. First oral tentacle compartment. CTO2. Second oral tentacle compartment. Di. Teeth. EO. Oral Shield. EAD. Adoral shield. Eead. Adoral shield spine. $2^{\circ}$ Eead. Second adoral shield spine. MEPO. Oral plate ridge spine. OLy. Lyman's ossicle. PaD. Dental papillae. PaI. Infradental papilla. PD. Dental plate. PGab. Abradial genital plate. PGad. Adradial genital plate. PO. Oral plate. $1^{\circ} \mathrm{PV}$. First ventral plate. 
los brazos delgadas, romas y largas, más largas que dos segmentos del brazo; las más dorsales aplanadas y cortas; el resto cónicas, siendo las medias las más largas. Las espinas del brazo más largas se encuentran cerca del segmento veinte. Dos poros tentaculares por segmento, cada uno con dos escamas tentaculares hasta el tercer segmento, una escama por cada poro en el resto del brazo. Pies ambulacrales digitiformes (Fig. 1G).

Coloración en alcohol, superficie del disco dorsal marrón claro, ventralmente color beige. Brazos marrón claro con bandas transversales marrón oscuro cada dos o tres placas dorsales; placas ventrales con línea vertical marrón claro a lo largo del brazo.

Descripción interna: Material general ICML-UNAM 10457. DD = $21.3 \mathrm{~mm}$. Vértebra con articulación de tipo zygospondylous. Vértebra distal (Fig. 3A) con SA en forma de "U". Cuatro FA ovaladas, con estereoma compacto. SM corta, con estereoma compacto. PRM ovalado, con estereoma con poros pequeños. $\mathrm{MuO}$ elevada, con estereoma compacto; MuA deprimida, con estereoma compacto. Vértebra proximal (Fig. 3B) con cuatro FA ovaladas, estereoma compacto. IM ovalada, con estereoma con poros grandes. $\mathrm{N}$ a cada lado de las FA ventrales. MuA elevada y $\mathrm{MuO}$ deprimida.

Placa dorsal de los brazos en vista externa casi dos veces más ancha que larga; extremo proximal recto con dos depresiones en cada extremo, extremo lateral recto; extremo distal redondeado con una depresión media cóncava (Fig. 3C). Estereoma con microlentes (364 en $0.5 \mathrm{~mm}^{2}$ ) distribuidos uniformemente en la superficie de la placa dorsal, rodeados de poros (Fig. 3E). Placa dorsal de los brazos en vista interna (Fig. 3D) con un canal en extremo proximal que se extiende hasta la mitad de la placa; $\mathrm{N}$ a cada lado de la placa. Estereoma poroso; hacia el extremo distal los poros son más pequeños.

Placa ventral de los brazos en vista externa (Fig. 3F) pentagonal, casi igual de ancha que larga; extremo proximal con ápice, el cual generalmente está cubierto por la placa anterior; bordes laterales casi rectos; extremo distal convexo. Estereoma con poros uniformes. Placa ventral de los brazos en vista interna (Fig. 3G) con un CR desde el ápice hasta el centro de la placa y una $\mathrm{N}$ a cada lado de la placa; extremo distal con FA largas, en forma gota y protuberantes. Estereoma poroso; hacia el extremo medio-distal los poros son más pequeños.

Escama tentacular en vista externa (Fig. $3 \mathrm{H})$ ovalada, estereoma poroso, con poros más pequeños hacia la inserción con la placa lateral. Escama tentacular en vista interna (Fig. 3I) con un pequeño $\mathrm{CO}$ redondeado con estereoma compacto; el resto de la escama tentacular con estereoma poroso.

Placa lateral de los brazos en vista externa (Fig. 3J) con forma de "C", más larga que ancha; terminación dorsal con un $\mathrm{CO}$, terminación ventral larga y algo aplanada. Siete AES con forma de voluta y dos perforaciones, una grande para la penetración muscular y otra pequeña para el nervio, separadas por un pliegue sigmoideo (Fig. 3L). Estereoma poroso, articulaciones con estereoma compacto. Placa lateral de los brazos en vista interna (Fig. 3K) con un $\mathrm{CO}$ en la terminación dorsal, terminación ventral con una $\mathrm{N}$ y dos FA. Espina del brazo robusta y roma (Fig. 3M), con estereoma con poros uniformes (Fig. $3 \mathrm{~N}$ ).

Gránulos del disco ovalados de apariencia espiniforme, base redonda con poros grandes y uniformes (Fig. 3O, Fig. 3P, Fig. 3Q).

EO en vista externa (Fig. 4A) casi redondo, proximalmente truncado; distalmente con una muesca. Estereoma poroso con perforaciones uniformes. EO en vista interna (Fig. 4B) con cuatro FA protuberantes, las proximales redondas y las distales semi-ovaladas. Bordes laterales deprimidos; en medio un canal ligeramente deprimido. Estereoma poroso.

EAD en vista externa (Fig. 4C) con forma de paralelogramo, más largo que ancho; todos sus bordes cóncavos, bordes laterales deprimidos. Estereoma con poros pequeños adradialmente, abradialmente con poros más grandes. Tres N, una FA redonda en el extremo distal. EAD en vista interna (Fig. 4D) con una FA 


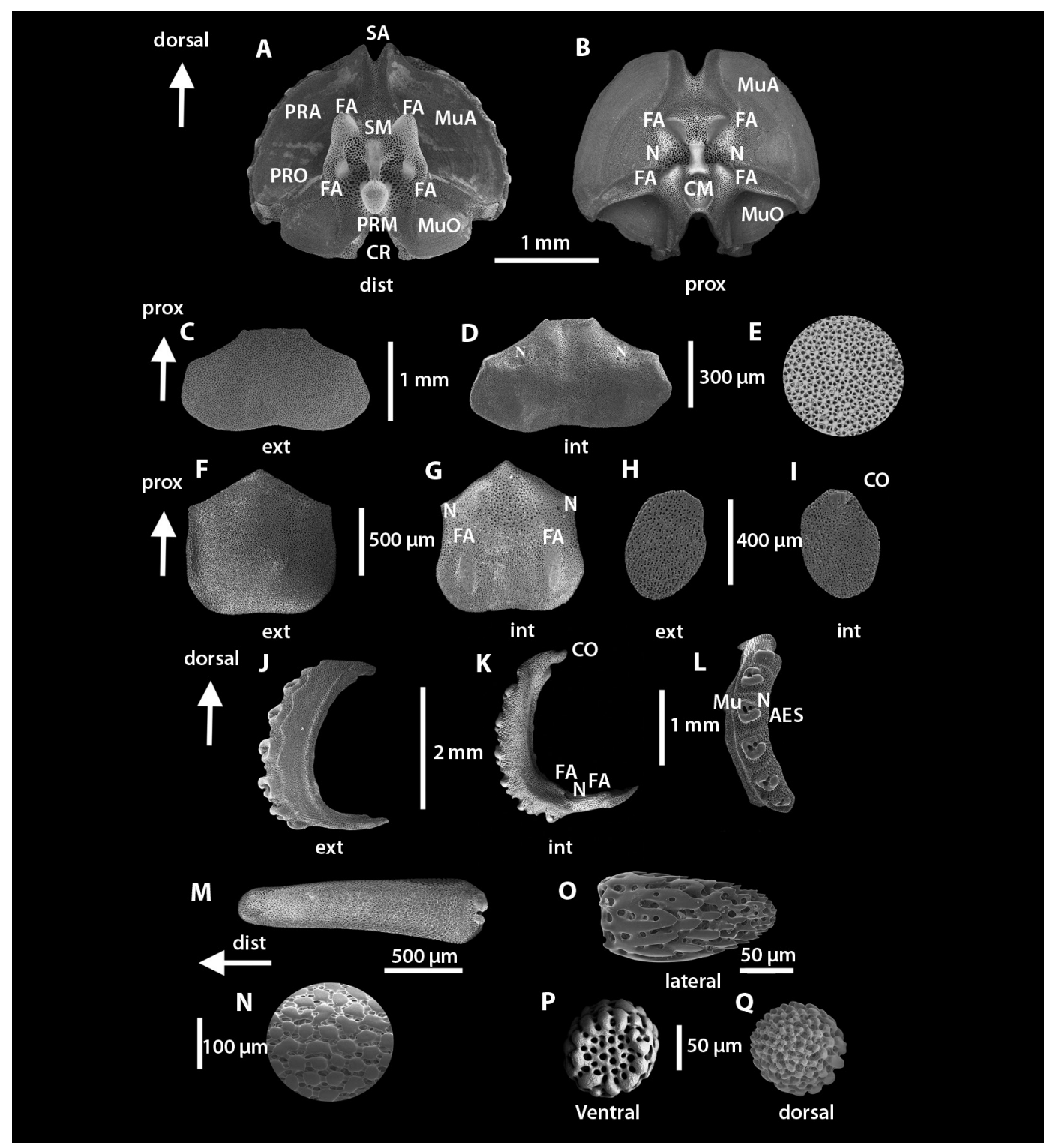

Fig. 3. Ophiocomella alexandri. ICML-UNAM 10457. DD = $21.3 \mathrm{~mm}$. Microestructuras internas. A-B. Vértebra. C-D. Placa dorsal del brazo. E. Microlentes de la placa dorsal externa. F-G. Placa ventral del brazo. H-I. Escama tentacular. J-K. Placa lateral del brazo. L. Articulación de las espinas de los brazos. M. Espina del brazo. N. Estereoma de la espina del brazo. O-Q. Gránulos del disco. AES. Articulación de la espina. CR. Canal radial. CO. Cóndilo. CM. Cuenca media. FA. Faceta articulada. Mu. Perforación muscular. MuA. Área muscular aboral. MuO. Área muscular oral. N. Perforación nerviosa. PRA. Proceso aboral. PRM. Proceso medio. PRO. Proceso oral.

Fig. 3. Ophiocomella alexandri. ICML-UNAM 10457. DD $=21.3 \mathrm{~mm}$. Internal microstructures of the arm. A-B. Vertebrae. C-D. Dorsal arm plate. E. Microlenses of the external dorsal plate. F-G. ventral arm plate. H-I. Tentacle scale. J-K. Lateral arm plate. L. Spine articulation. M. Arm spine. N. Arm spine stereoma. O-Q Disc granules. AES. Spine articulation. CR. Radial channel. CO. Condyle. CM. Medium insertion. FA. Articular surface. Mu. Muscle perforation. MuA. Aboral muscular area. MuO. Oral muscle area. N. Nerve perforation. PRA. Aboral process. PRM. Medium process. PRO. Oral process. 
pronunciada en el extremo distal del borde adradial, $\mathrm{N}$ a la mitad de la placa.

PO más largas que anchas y robustas. Vista adradial de la PO (Fig. 3E) con Mad pequeña; AAad elevada, prominente y con surcos profundos. Dos compartimientos de tentáculos orales, redondos, uno dorsal (CTO1) y otro ventral (CTO2) (Fig. 4E). MEPO elevada con estereoma compacto. Vista abradial de la $\mathrm{PO}$ alta (Fig. 4F). Mab con forma de riñón y con estrías transversales marcadas; extremo proximal con surcos laberínticos, dorsalmente se encuentra el CR. Estereoma poroso en ambas vistas, áreas musculares con estereoma compacto.

Placa dental en vista externa (Fig. 4G) en una sola pieza, rectangular con bordes redondeados. Tres forámenes perforados, ovalados, divididos longitudinalmente por un $\mathrm{S}$ y rodeadas por FA prominentes; un foramen no perforado y 15 inserciones papilares redondas. Estereoma poroso, en las FA es compacto. Placa dental en vista interna (Fig. 4H) con perforaciones divididas por $\mathrm{S}$ y flanqueadas por FA en forma de líneas verticales que se extienden desde el extremo dorsal hasta la mitad de la placa. Seis FA en forma de líneas horizontales desde la mitad de la placa hasta el extremo ventral.

Cuatro Di, los más dorsales cuadrangulares (Fig. 4I, Fig. 4J) y los más ventrales triangulares (Fig. 4K, Fig. 4L). Estereoma compuesto, parte proximal compacta y parte distal porosa.

PGab (Fig. 4M) de menor tamaño que la placa adradial, ligeramente arqueada. Extremo proximal redondeado y aplanado; extremo distal con una cabeza que articula con la placa adradial (Fig. 2C, Fig. 2B). Estereoma poroso dorsalmente y más compacto ventralmente. PGad (Fig. 4N) con más volumen que la placa abradial. Extremo proximal redondeado, a lo largo de la placa hay un canal cóncavo que termina hasta el extremo distal donde hay una N. Extremo distal con una depresión y un $\mathrm{CO}$ (Fig. 4C); AL al centro, en el extremo ventral estereoma poroso.

ER en vista externa (Fig. 4O) en forma de bota, más largo que ancho; extremo proximal con terminación redondeada y extremo distal con surco no muy pronunciado en la parte media. Estereoma poroso; hacia los bordes los poros son más pequeños. ER en vista interna (Fig. 4P) con un $\mathrm{CO}$ ovalado y dos $\mathrm{N}$ en el extremo distal.

Variaciones: Con base en la revisión de material general y de los ejemplares tipo, se observaron diferencias en la forma del disco. Los ejemplares de DD $<20 \mathrm{~mm}$ presentaron con mayor frecuencia disco circular, mientras que en ejemplares de $\mathrm{DD}=2-6 \mathrm{~mm}$ fue más recurrente la forma pentagonal.

En los brazos, las vértebras de los ejemplares de DD > $10 \mathrm{~mm}$ presentaron variación en la forma del PM que puede ser redondo, ovalado o triangular con bordes redondeados; por lo tanto, la CM presenta las mismas variaciones. En ejemplares de DD = 2-6 mm las vértebras proximales son más largas que en organismos de mayor tamaño, en vista proximal la IM no está muy bien definida; se asemeja a un triángulo con bordes redondeados. El estereoma del área muscular proximal y distal es más poroso en ejemplares pequeños $(\mathrm{DD}=2-6 \mathrm{~mm}$ ) en comparación con el de los grandes (DD $>10$ $\mathrm{mm}$ ). En vista distal, la sutura media de la vértebra es más visible en ejemplares pequeños (Fig. 5A, Fig. 5B).

En las placas dorsales de los brazos, algunos ejemplares grandes (DD $>10 \mathrm{~mm}$ ) tienen placas acorazonadas y otras ovaladas (ICMLUNAM 3.9.114), a su vez, el ancho del extremo proximal es variable. En ejemplares pequeños $(\mathrm{DD}=2-6 \mathrm{~mm}$ ) las placas dorsales de los brazos tienen forma de abanico y son casi igual de largas que anchas; el estereoma es poroso y con algunos microlentes definidos (Fig. 5C).

Las placas ventrales de los brazos en los ejemplares pequeños $(\mathrm{DD}=2-6 \mathrm{~mm})$ conservan la forma pentagonal, aunque son más largas que anchas; el estereoma es compacto con poros pequeños. En vista interna el estereoma de las FA no es tan compacto en comparación con los ejemplares grandes (DD > $10 \mathrm{~mm}$ ) (Fig. 5D).

El número de escamas tentaculares varió de dos a tres por poro tentacular, las cuales se 


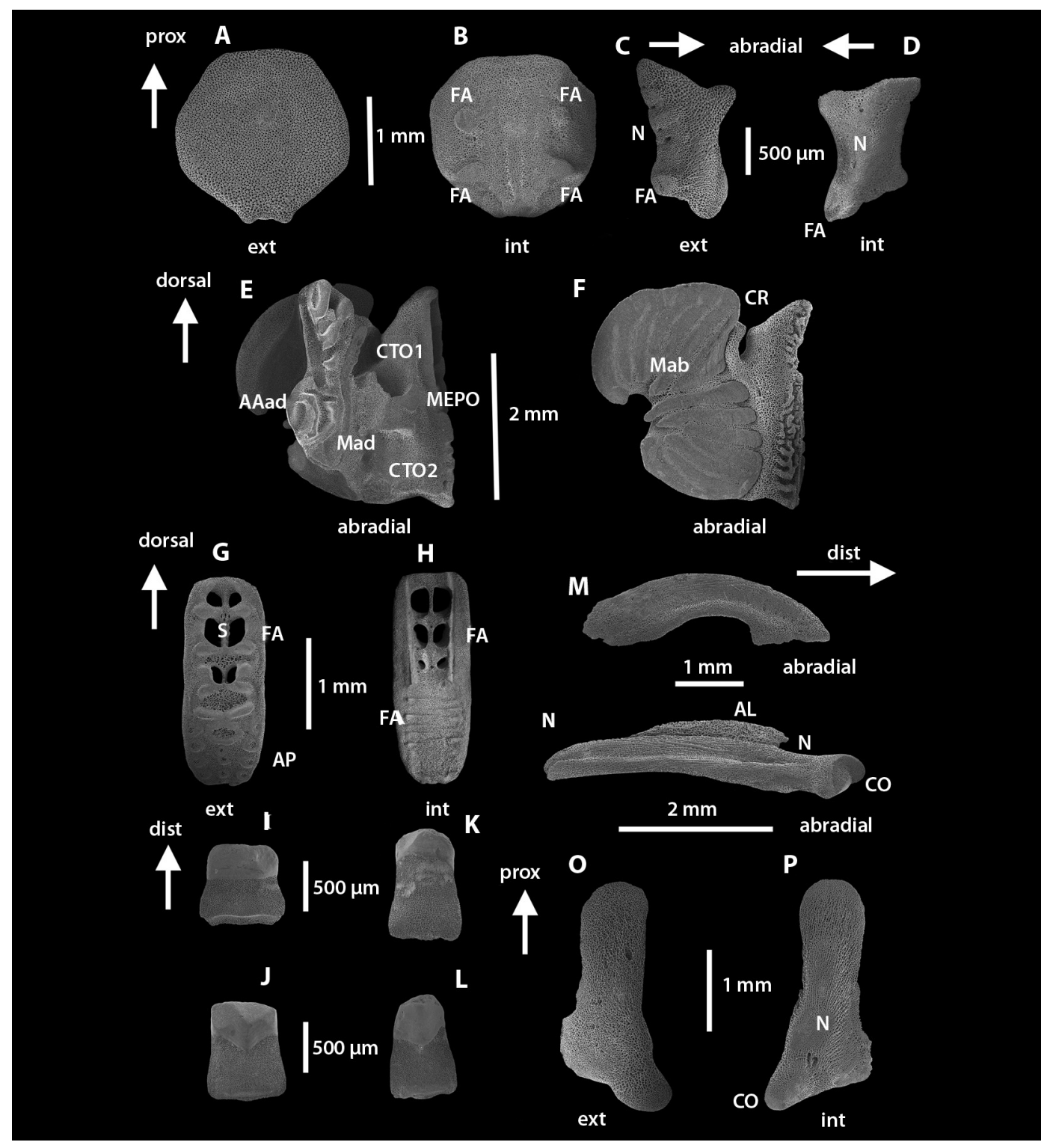

Fig. 4. Ophiocomella alexandri. ICML-UNAM 10457. DD $=21.3 \mathrm{~mm}$. Microestructuras internas. A-B. Escudo oral. C-D. Escudo adoral. E-F. Placa oral. G-H. Placa dental. I-J. Dientes dorsales. K-L. Dientes ventrales. M-N. Placas genitales. O-P. Escudo radial. AAad. Área de articulación adradial. AL. Aleta. AP. Área papilar. MEPO. Meseta de la espina de la placa oral. CN. Canal nervioso. CO. Cóndilo. CTO1. Primer compartimiento del tentáculo oral. CTO2. Segundo compartimiento del tentáculo oral. FA. Faceta articulada. Mad. Área muscular adradial. Mab. Área muscular abradial. N. Perforación nerviosa. S. septum.

Fig. 4. Ophiocomella alexandri. ICML-UNAM 10457. DD $=21.3 \mathrm{~mm}$. Internal microstructures. A-B. Oral shield. C-D. Adoral shield. E-F. Oral plate. G-H. Dental plate. I-J. Dorsal teeth. K-L. Ventral teeth. M-N. Genital plates. O-P. Radial shield. AAad. Adradial joint area. AL. Fin. AP. Papillary area. FA. Articular surface. MEPO. Oral plate ridge spine. CR. Radial channel. CO. Condyle. CTO1. First oral tentacle compartment. CTO2. Second oral tentacle compartment. Mad. Adradial muscle area. Mab. Abradial muscular area. N. Nerve perforation. S. Septum. 


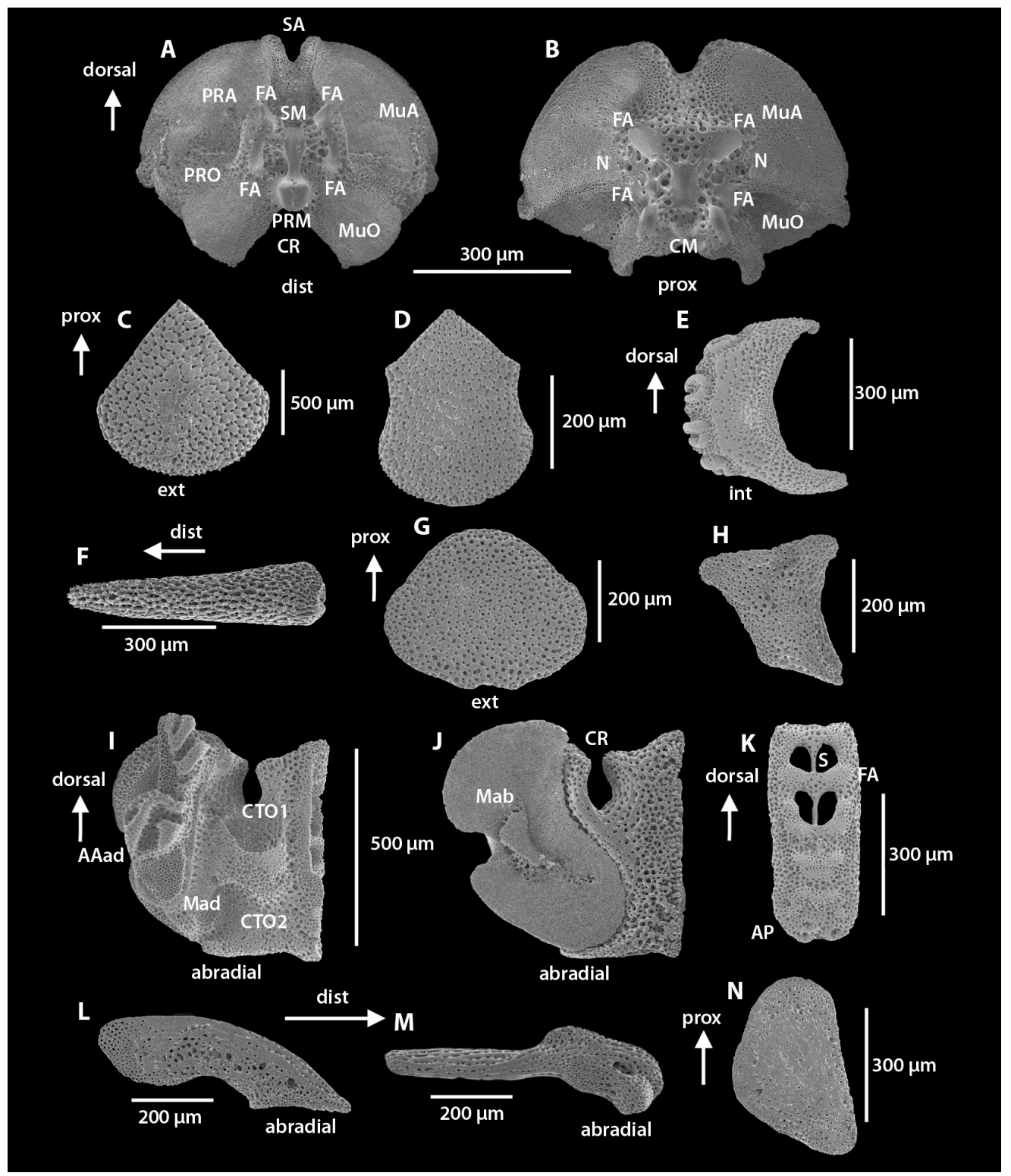

Fig. 5. Ophiocomella alexandri. ICML-UNAM 10457. DD = $3.12 \mathrm{~mm}$. Microestructuras internas. A-B. Vértebra. C. Placa dorsal del brazo. D. Placa ventral del brazo. E. Placa lateral del brazo. F. Espina del brazo. G. Escudo oral. H. Escudo adoral. I-J. Placa oral. K. Placa dental. L-M. Placas genitales. N. Escudo radial. AAad. Área de articulación adradial. CM. Cuenca media. CR. Canal radial. CTO1. Primer compartimiento del tentáculo oral. CTO2. segundo compartimiento del tentáculo oral. FA. Faceta articulada. MEPO. Meseta de la espina de la placa oral. Mab. Área muscular abradial. Mad. Área muscular adradial. MuA. Área muscular aboral. MuO. Área muscular oral. N. Perforación nerviosa. PRA. Proceso aboral. PRM. Proceso medio. PRO. Proceso oral. S. Septum.

Fig. 5. Ophiocomella alexandri. ICML-UNAM 10457. DD $=3.12 \mathrm{~mm}$. Internal microstructures of the arm. A-B. Vertebrae. C. Arm dorsal plate. D. Arm ventral plate. E. Arm lateral plate. F. Arm spine. G. Oral shield. H. Adoral shield. I-J. Oral plate. K. Dental L-M. Genital plates. N. Radial shield. AAad. Adradial joint area. CM. Medium insertion. CR. Radial channel. CTO1. First oral tentacle compartment. CTO2. Second oral tentacle compartment. FA. Articular surface. MEPO. Oral plate ridge spine. Mab. Abradial muscular area. Mad. Adradial muscle area. MuA. Aboral muscular area. MuO. Oral muscle area. N. Nerve perforation. PRA. Aboral process. PRM. Medium process. PRO. Oral process. S. Septum. 
van reduciendo en número hacia el extremo distal, y pueden estar presentes hasta el octavo segmento. En los brazos de un mismo ejemplar también se encuentran estas variaciones (USNM 38906).

Las placas laterales en ejemplares pequeños $(\mathrm{DD}=2-6 \mathrm{~mm}$ ) tienen un arco menos pronunciado, extremos dorsal y ventral no tan prolongados en comparación con los ejemplares grandes (DD $>10 \mathrm{~mm})$. El estereoma de los ejemplares pequeños $(\mathrm{DD}=2-6 \mathrm{~mm})$ que está cerca de las inserciones es compacto con escasos poros pequeños (Fig. 5E).

El número de espinas de los brazos en ejemplares pequeños (DD $=2-6 \mathrm{~mm}$ ) no es de más de cuatro espinas, mientras que en ejemplares grandes (DD $<10 \mathrm{~mm}$ ) pueden ser siete. El estereoma en los ejemplares pequeños $(\mathrm{DD}=2-6 \mathrm{~mm})$ tiene poros más grandes en comparación con los ejemplares grandes (DD $>10 \mathrm{~mm}$ ) (Fig. 5F).

En la mandíbula, el OLy puede ser aserrado (ICML-UNAM 3.9.12). Los EO, aunque generalmente son redondos, en algunos casos pueden ser más anchos que largos (USNM E14024), o bien, más largos que anchos (ICMLUNAM 3.9.61). Los ejemplares pequeños (DD $=2-6 \mathrm{~mm}$ ) con mayor frecuencia tienen un EO más ancho que largo y un estereoma con poros más pequeños (Fig. $5 \mathrm{G}$ ).

Los EAD de los ejemplares grandes (DD $>10 \mathrm{~mm}$ ) presentaron variación en la amplitud del extremo proximal. En ejemplares pequeños $(\mathrm{DD}=2-6 \mathrm{~mm}$ ) los escudos adorales son muy estrechos distalmente (Fig. $5 \mathrm{H}$ ).

En las PO, la vista adradial de los ejemplares pequeños $(\mathrm{DD}=2-6 \mathrm{~mm}$ ) tienen los surcos laberínticos de la unión con la siguiente mandíbula bien definidos; sin embargo, el estereoma tiene poros grandes (Fig. 5I). En la vista abradial de los ejemplares grandes de DD $>20$ $\mathrm{mm}$ presentaron una mayor extensión del Mab que en los ejemplares de DD = $10 \mathrm{~mm}$. El Mab en ejemplares pequeños ( $\mathrm{DD}=3-6 \mathrm{~mm}$ ) está reducida y no presenta las estrías, si no un estereoma con poros pequeños y juntos (Fig. 5J).

Las $\mathrm{PaD}$ de los ejemplares grandes (DD $>$ $10 \mathrm{~mm}$ ) pueden llegar a ser hasta 16 en número, distribuidas en tres o cuatro líneas verticales (ICML-UNAM 3.9.21); en cambio, los ejemplares pequeños $(\mathrm{DD}=2-6 \mathrm{~mm})$ presentan de cuatro a seis $\mathrm{PaD}$ distribuidas en dos líneas verticales (ICML-UNAM 10457).

La placa dental de los ejemplares grandes (DD $>10 \mathrm{~mm}$ ) tuvo variación en el número de forámenes que va de tres a cuatro, de los cuales, pueden ser dos o tres perforados. En ejemplares de DD $=20 \mathrm{~mm}$ el AP puede ser más extensa. En los ejemplares pequeños (DD = 2-6 mm) el AP está reducida, con solo cinco inserciones de papilas, el estereoma de las FA que rodean los forámenes tiene pequeños poros muy separados (Fig. 5K). En ejemplares más pequeños $(\mathrm{DD}=1 \mathrm{~mm})$ el AP no es visible.

Las PGab y PGad en ejemplares grandes (DD $>10 \mathrm{~mm}$ ) presentaron variación en su longitud. En ejemplares pequeños (DD $=2-6$ $\mathrm{mm}$ ) la AL de la PGad es muy corta y reducida. El estereoma es compacto con pocos poros pequeños (Fig. 5L, Fig. 5M)

En ejemplares intactos, los ER siempre están cubiertos por granulación y no son visibles, a pesar de ello, en algunos ejemplares se observaron con forma de bota (ICML-UNAM 3.9.97). Los ejemplares grandes ( $\mathrm{DD}>10 \mathrm{~mm}$ ) mostraron variación en el ancho y largo del ER $y$ en el surco presente en el extremo distal, el cual puede ser más pronunciado. En ejemplares pequeños (DD = 2-6 mm) los ER tienen forma triangular con bordes redondeados y el estereoma tiene poros pequeños y separados que se van haciendo más grandes hacia los bordes (Fig. $5 \mathrm{~N}$ ).

Respecto a la coloración, se observó una amplia gama en el disco: amarillo claro, amarillo con manchas marrones, amarillo verdoso, amarillo grisáceo, marrón claro, marrón con manchas amarillas, marrón rojizo y marrón verdoso. Es importante señalar que los colores más claros generalmente son más recurrentes en los ejemplares preservados en alcohol etílico (70 \%) debido a la decoloración causada por el alcohol. No se observó un patrón de coloración definido para alguna área geográfica específica. En cuanto a la coloración de las placas dorsales 


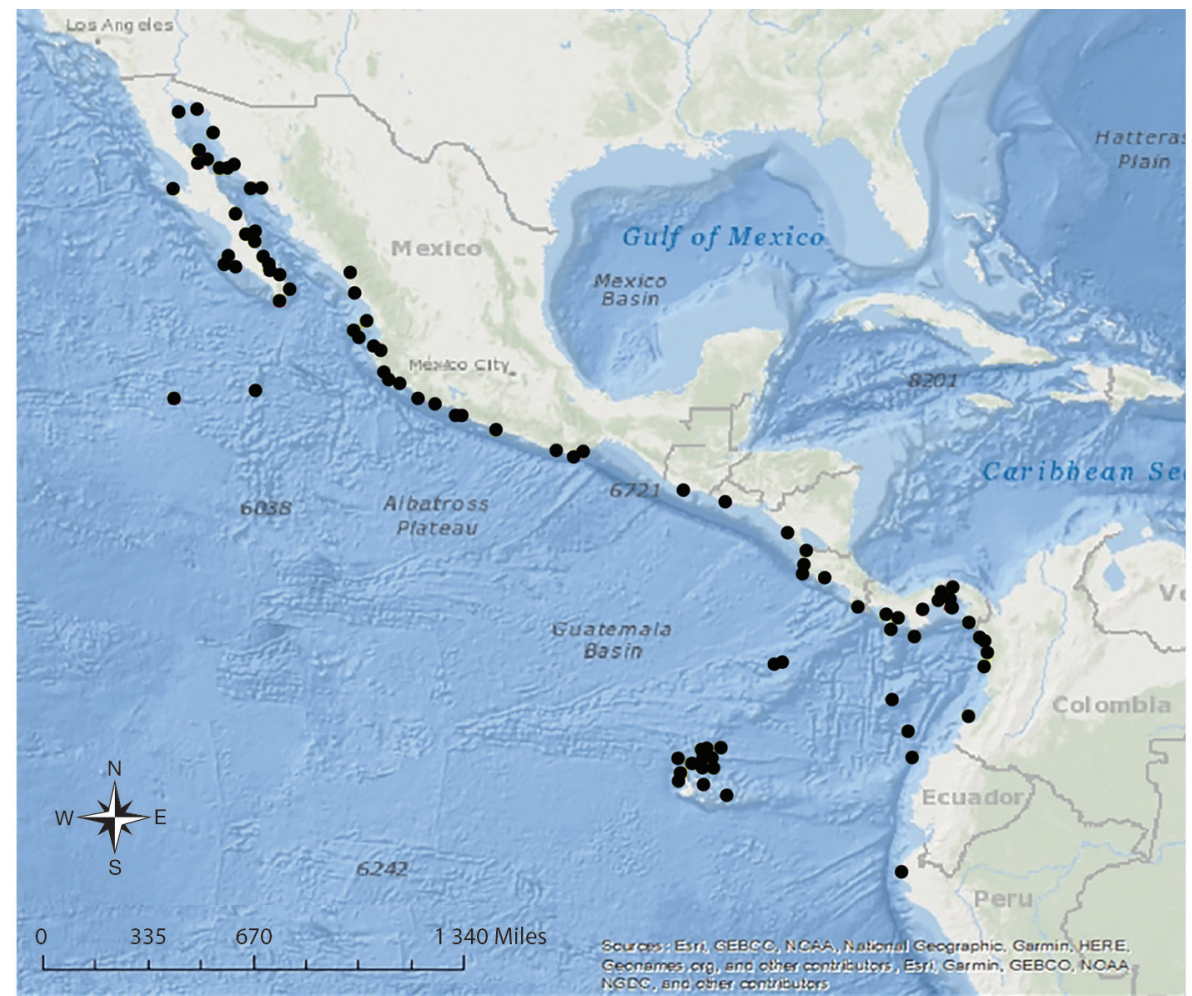

Fig. 6. Distribución geográfica de Ophiocomella alexandri.

Fig. 6. Geographical distribution of Ophiocomella alexandri.

de los brazos, éstas pueden presentar manchas marrones o beige, con aspecto marmoleado.

Distribución geográfica: Pacífico Oriental Tropical: México (incluyendo islas del golfo de California, islas Revillagigedo e islas Marías), Guatemala, El Salvador, Nicaragua, Costa Rica (incluyendo a la isla del Coco), Panamá, Colombia (incluyendo isla Malpelo e isla Gorgona), Ecuador (incluyendo islas Galápagos) y Perú (incluyendo islas Lobos de Afuera) (Granja Fernández et. al., 2014; Granja-Fernández \& Hooker, 2020) (Fig. 6).

Distribución batimétrica: Habita desde la zona intermareal hasta los $57 \mathrm{~m}$ (USNM 39349).

Hábitat: Se puede encontrar en costas rocosas y arrecifes de coral, aunque también se ha observado en algas y rodolitos (GranjaFernández et al., 2014).

\section{DISCUSIÓN}

Ophiocomella alexandri es una especie conspicua del POT, y a pesar de ello no se ha documentado su variación morfológica. Hasta el momento las descripciones de la especie han estado restringidas a organismos adultos $\mathrm{y}$ han sido inconsistentes respecto a la forma y número de papilas asociadas a la mandíbula. $\mathrm{La}$ descripción presentada en este trabajo detalla las variaciones en los caracteres morfológicos externos e internos respecto al tamaño, actualizando la descripción de la especie bajo la nueva terminología propuesta (Hendler, 2018), siendo la más completa hasta el momento. Se 
delimitaron los caracteres taxonómicos diagnósticos variables (papilas asociadas a la mandíbula, forma de las placas dorsales de los brazos y escamas tentaculares) y se exhiben por primera vez las microestructuras internas de importancia taxonómica de la mandíbula y del brazo.

Existe una gran variación en la coloración de $O$. alexandri la cual ya había sido reportada por Granja-Fernández et al. (2014). De acuerdo a diversos autores (Hendler, 1984; O’Hara, Byrne, \& Cisternas, 2004) esta variación se debe a la capacidad especial que tienen las especies de la familia Ophiocomidae de cambiar de color en respuesta a la luz y como mecanismo de defensa. Por lo anterior, la coloración puede estar influenciada por el método y hora de captura; así como otros factores como el tipo y tiempo de preservación. A pesar de ello, en el presente trabajo se encontró que las coloraciones verdosas son más recurrentes al norte de su área de distribución $\left(32^{\circ} \mathrm{N}-23^{\circ} \mathrm{N}\right)$, mientras que hacia la zona centro y sur, la coloración tiende a ser más amarillenta-marrón $\left(23^{\circ} \mathrm{N}-6^{\circ} \mathrm{S}\right)$. Esto último coincide con la caracterización de aguas templadas al norte y agua tropicales al centro y sur del POT propuesta por Spalding et al. (2007). Sin embargo, es necesario hacer un registro de las coloraciones in vivo para conocer si esta correspondencia entre coloración es acertada.

En relación a la morfología de los brazos, las variaciones están principalmente relacionadas al tamaño de los ejemplares. Entre los ejemplares de DD > $10 \mathrm{~mm}$, existe una variación en la forma de las placas dorsales de los brazos. De acuerdo con la diagnosis original de la especie (Lyman, 1860), las placas cordiformes son las que caracterizan a $O$. alexandri; $\sin$ embargo, se encontraron frecuentemente placas ovaladas en ejemplares de DD $<20 \mathrm{~mm}$. Este tipo de placas ovaladas tienen forma similar a las placas distales, lo que sugiere que se siguen agregando segmentos al brazo (H.L. Clark, 1914). Martínez-Morales (2019) señala que el DD en la primera madurez sexual es de 13.7 $\mathrm{mm}$, de acuerdo con este valor, los ejemplares a partir de esta talla podrían caracterizarse como adultos; sin embargo, se siguen añandiendo segmentos a sus brazos. Aunado a esto, también se observaron variaciones en el número de articulaciones de espinas de las placas laterales. A pesar de que $O$. alexandri muestra un patrón ascendente en el número de espinas, particularmente para el segmento en estudio (quinto segmento del brazo), la variación fue de cinco a siete espinas, indicando que los segmentos más jóvenes tendrán menor número de espinas (Stöhr, 2005).

Los microlentes observados en las placas dorsales de los brazos habían sido reportados con anterioridad para especies de la familia Ophiocomidae pero no para $O$. alexandri (Sumner-Rooney, Rahman, Sigwart \& UllrichLüter, 2018). Estos microlentes o trabéculas periféricas expandidas (EPT) están asociados a paquetes de fibras nerviosas y cromatóforos; formando un sistema fotorreceptor (Hendler \& Byrne, 1987) y en los últimos años se han realizado pruebas de inmunohistoquímica, histología y microtomografía de sincrotrón para conocer la función de dichas estructuras (Sumner-Rooney et al., 2018; SumnerRooney, Kirwan, Lowe \& Ullrich-Lüter, 2020). Sumner-Rooney et al. (2020) reconstruyeron modelos de los fotorreceptores de Ophiomastix wendtii (Müller \& Troschel, 1842) y Ophiocomella pumila (Lütken, 1856) (anteriormente clasificadas bajo el género Ophiocoma), donde concluyeron que aunque las dos especies tienen EPT esféricos en las placas dorsales de los brazos, solo $O$. wendtii posee una visión extraocular producida por los cromatóforos. En $O$. pumila no se observaron estos cromatóforos, lo que explica una insensibilidad a la luz y en consecuencia no cambia tan drásticamente de color en comparación con $O$. wendtii. Con base en lo anterior y de acuerdo a la nueva clasificación de la familia (O'Hara et al., 2019), a pesar que $O$. alexandri también posee EPT, esta visión extraocular parece estar restringida al género Ophiomastix (Sumner-Rooney et al., 2018) por lo que es necesario continuar con estudios al respecto en otras especies pertenecientes a la familia, ya que podría considerarse un caracter de importancia taxonómica. De acuerdo con 
Sumner-Rooney et al. (2020) dentro de la familia Ophiocomidae, los cromatóforos son un caracter derivado y en los EPT pueden cumplir ancestralmente una tarea no visual pero vital, como evitar la exposición a la luz, como en el caso $O$. pumila congénere de $O$. alexandri.

El número de escamas tentaculares se ha empleado como un caracter diagnóstico para $O$. alexandri, diferenciándola de quien fuera su congénere Ophiocoma aethiops. En esta revisión, la extensión del par de escamas tentaculares no fue correspondiente a lo señalado en la diagnosis (Lyman, 1860), ni fue constante en todos los brazos de un mismo individuo. H.L. Clark (1921) señaló que este caracter es de importancia taxonómica; sin embargo, consideró su variabilidad como un caso de reversión, por lo cual es difícil emplearlo como caracter específico válido para la familia Ophiocomidae.

Respecto a las estructuras de la mandíbula, el número de papilas orales reportadas ha sido variable (Caso, 1951; Granja-Fernández et al., 2014). O. alexandri posee un conglomerado de $\mathrm{PaD}$ en el ápice de la mandíbula, por lo que en muchas ocasiones estas papilas fueron consideradas papilas orales. En la descripción original, Lyman (1860) señaló que $O$. alexandri posee de nueve a 10 papilas orales; sin embargo, bajo la nueva terminología de las papilas asociadas sensu lato (Hendler, 2018) esta especie posee solo ocho. El número de papilas orales es un caracter conservado aún en los estadios juveniles (Stöhr, 2005); sin embargo, deben estudiarse con cuidado ya que éstas van apareciendo gradualmente durante la ontogenia (Hendler, 2018). El describir a las especies bajo esta terminología propuesta por Hendler (2018) facilitará su correcta identificación. Por otro lado, las $\mathrm{PaD}$ también presentan una gran variación en número (de cuatro a 16), tanto en lo reportado (Nielsen, 1932; Caso, 1951; Cortés-Fernández, 1999; Conejeros-Vargas, 2015) como en lo observado en el presente trabajo; esta variación está relacionada con el tamaño de los ejemplares. En contraste con las papilas orales, las $\mathrm{PaD}$ siguen añadiéndose, aún siendo ejemplares de DD $>20 \mathrm{~mm}$, llegando a ser hasta 16. En la parte más ventral de la placa dental se observan papilas dentales más pequeñas respecto al resto, que de acuerdo con Hendler (2018), corresponde a papilas jóvenes.

$\mathrm{El}$ número de $\mathrm{PaD}$ está relacionado con la extensión de la placa dental. La placa dental se ha empleado para separar grupos taxónomicos (Devaney, 1970; Benavides-Serrato \& O'Hara, 2008) basándose principalmente en su longitud, amplitud y forámenes perforados; sin embargo, la variación intraespecifíca aún es desconocida. En este trabajo se muestran las variaciones en $O$. alexandri, tanto en el AP como en el número de forámenes perforados que por el momento, se sugiere que está relacionado al tamaño y por lo tanto, a su estrategia alimentaria. Hendler (2018), mencionó que un menor número de papilas dentales en organismos juveniles (por lo tanto una placa dental más corta) limita la alimentación por material en suspensión, en donde las papilas dentales tienen una función importante. Esta observación fue correspondiente a lo observado en este trabajo donde los ejemplares de DD =2-6 $\mathrm{mm}$ presentaron solo cuatro papilas dentales en comparación con ejemplares mayores de $\mathrm{DD}>20 \mathrm{~mm}$.

Es necesario realizar esfuerzos para la descripción morfológica interna y externa de las especies que integran la familia Ophiocomidae. Dados los recientes cambios señalados por O'Hara et al. (2019) a través de evidencias moleculares, no deja de ser importante definir los caracteres morfológicos y sus variantes para poder delimitar a los géneros y especies que pertenecen a esta familia con grandes problemas taxonómicos.

Declaración de ética: los autores declaran que todos están de acuerdo con esta publicación y que han hecho aportes que justifican su autoría; que no hay conflicto de interés de ningún tipo; y que han cumplido con todos los requisitos y procedimientos éticos y legales pertinentes. Todas las fuentes de financiamiento se detallan plena y claramente en la sección 
de agradecimientos. El respectivo documento legal firmado se encuentra en los archivos de la revista.

\section{AGRADECIMIENTOS}

Los autores agradecen a Alicia de la Luz Durán González (Laboratorio de Sistemática y Ecología de Equinodermos) por el acceso al material depositado en la ICML-UNAM. A Adam Baldinger (MCZ) por el acceso al material tipo. A Christopher Meyer (USNM) por el apoyo en la visita a la colección. A Scott Whittaker (USNM) por el soporte para la toma de fotografías multifocales. A Berenit Mendoza Garfias (Laboratorio de Microscopía y Fotografía de la Biodiversidad I, Instituto de Biología, UNAM) por la toma de fotografías de microscopía electrónica de barrido. A Magdalena De los Palos Peña y a Michel García Luna por la ayuda en la realización de los esquemas. GIRC (becario 492379) agradece al Posgrado de Ciencias del Mar y Limnología UNAM y al Consejo Nacional de Ciencia y Tecnología (CONACyT) por la beca de doctorado 281719.

\section{RESUMEN}

\section{Morfología y microestructura interna de la estrella quebradiza Ophiocomella alexandri (Echinodermata: Ophiocomidae)}

Introducción: Ophiocomella alexandri es una especie conspicua del Pacífico Tropical Oriental. La diagnosis esta basada en especimenes adultos y no existen variaciones documentadas. La inclusión de microestructuras internas a las descripciones permitirá hacer comparaciones intraespecíficas e interespecíficas en taxones con problemas taxonómicos. Objetivo: Describir la morfología externa y las microestructuras internas de $O$. alexandri, incluida la variación. Métodos: Revisamos el material tipo de $O$. alexandri y el material en general depositado en tres colecciones científicas. Examinamos las microestructuras internas del brazo y la mandíbula con un microscopio electrónico de barrido. Resultados: Se describió detalladamente la morfología externa e interna mostrando las variaciones morfológicas de acuerdo al tamaño. Conclusiones: Las variaciones de la morfología interna de $O$. alexandri muestran que es necesario este tipo de análisis, y más importante aún que la morfología externa. Se recomienda realizar mas trabajos taxonómicos que incluyan a otras especies del género para esclarecer su estado taxonómico.

Palabras clave: brazo, mandíbula, caracteres, variaciones, microscopía electrónica de barrido

\section{REFERENCIAS}

Anzo-Martínez, A.D. (1994). Ofiuroideos (Echinodermata: Ophiuroidea) de las playas Pedregosa y Manzanillo Municipio de Zihuatanejo, Guerrero, México (Tesis de licenciatura). Universidad Michoacana de San Nicolás de Hidalgo, México.

Benavides-Serrato, M. \& O’Hara, T.D. (2008). A new species in the Ophiocoma erinaceus complex from the South-west Pacific Ocean (Echinodermata: Ophiuroidea: Ophiocomidae). Memoirs of Museum Victoria, $65,51-56$.

Birkeland, C., Meyer, D.L., Stanes, J.P. \& Buford, C.L. (1975). Subtidal communities of Malpelo Island. Smithsonian Contribution to Zoology, 176, 55-68.

Boone, L. (1926). Scientific results of the first oceanographic expedition of the "Pawnee". Echinoderms from the Gulf of California and the Perlas Islands. Bulletin of the Bingham Oceanographic Collection, 2(6), 1-14.

Brusca, R.C. (1980). Common intertidal invertebrates of the Gulf of California. EUA: University of Arizona press.

Campbell, A.S. (1921). Ophiuroidea of the West coast of North America. Journal of Entomology and Zoology, $13,46-49$.

Caso, M.E. (1951). Contribución al conocimiento de los ofiuroideos de México. (1). Algunas especies de ofiuroideos litorales. Anales del Instituto de Biología, Universidad Nacional Autónoma de México, 22(1), 219-312.

Caso, M.E. (1961). Los equinodermos de México (Tesis doctoral). Universidad Nacional Autónoma de México, México.

Caso, M.E. (1979). Los equinodermos de la Bahía de Mazatlán, Sinaloa. Anales del Centro de Ciencias del Mar y Limnología, Universidad Nacional Autónoma de México, 6, 197-368.

Caso, M.E. (1992). Los Equinodermos (Asteroideos, Ofiuroideos y Equinoideos de la Bahía de Mazatlán, Sinaloa. Instituto de Ciencias del Mar y Limnología, Universidad Nacional Autónoma de México, 11, $1-214$.

Caso, M.E., Laguarda-Figueras, A., Solís-Marín, F.A., Ortega-Salas, A. \& Durán-González, A.L. (1996). Contribución al conocimiento de la ecología de las comunidades de equinodermos de la Bahía de 
Mazatlán, Sinaloa. México. Anales del Centro de Ciencias del Mar y Limnología, Universidad Nacional Autónoma de México, 22, 101-119.

Clark, H.L. (1913). Echinoderms from Lower California, with descriptions of new species. Bulletin of the American Museum of Natural History, 32, 185-236.

Clark, H.L. (1915). Catalogue of recent Ophiurans, based on the collections of the Museum of Comparative Zoology. Memoirs of the Musuem of Comparative Zoology, 25(4), 165-376.

Clark, H.L. (1917). Hawaiian and other Pacific Echini. Memoirs of the Museum of Comparative Zoology, $46,85-283$

Clark, H.L. (1921). The Echinoderm Fauna of Torres Strait: Its Composition and Its Origin. Carnegie Institution of Washington Publication, 294, 1-223.

Clark, A.H. (1922). The ophiurans of the island of Curaçao. Bijdragen tot de Dierkunde, 22, 209-213.

Clark, H. L. (1923). Echinoderms from Lower California, with descriptions of new species: supplementary report. Bulletin of the American Museum of Natural History, 48(6), 147-163.

Clark, A.H. (1939). Echinoderms (other than holothurians) collected on the Presidential Cruise of 1938. Smithsonian Miscellaneous Collections, 98(11), 1-18.

Clark, H.L. (1940). Notes on Echinoderms from the West Coast of Central America. Eastern Pacific Expeditions of the New York Zoological Society. Zoological, $25,331-352$

Clark, H.L. (1942). The echinoderm fauna of Bermuda. Bulletin of The Museum of Comparative Zoology, 89, 367-391.

Conejeros-Vargas, C.A. (2015). Taxonomía y distribución de los equinodermos (Echinodermata) de las costas de Michoacán, México (Tesis de licenciatura). Universidad Nacional Autónoma de México, México.

Devaney, D.M. (1970). Studies on ophiocomid brittlestars. (1). A new genus (Clarkcoma) of Ophiocominae with a reevaluation of the genus Ophiocoma. Smithsonian Contributions to Zoology, 51, 1-41.

Downey, M.E. (1969). Catalog of recent ophiuroid type specimens in major collections in the United States. United States National Museum Bulletin, 293, 1-239.

Granja-Fernández, M.R. (2010). Los ofiuroideos (Echinodermata: Ophiuroidea) de Oaxaca, México (Tesis de licenciatura). Universidad del Mar, México.

Granja-Fernández, M.R. \& Hooker, Y. (2020). Revisiting the diversity and distribution of the ophiuroids (Echinodermata: Ophiuroidea) from Peru. Zootaxa, 4766(4), 539-556.
Granja-Fernández, R., Herrero-Pérezrul, M.D., LópezPérez, R.A., Hernández, L., Rodríguez-Zaragoza, F.A., Jones, R.W. \& Pineda-López, R. (2014). Ophiuroidea (Echinodermata) from coral reefs in the Mexican Pacific. ZooKeys, 406, 101-145.

Gray, J.E. (1840). A synopsis of the genera and species of the Class Hypostoma (Asterias Linnaeus). Annals and Magazine of Natural History, 6, 175-184.

Hendler, G. (1984). Brittlestar color-change and phototaxis (Echinodermata: Ophiuroidea: Ophiocomidae). Marine Ecology, 5(4), 379-401.

Hendler, G. (2018). Armed to the teeth: a new paradigm for the buccal skeleton of brittle stars (Echinodermata: Ophiuroidea). Contributions in Science, 526, 189-311.

Hendler, G. \& Byrne, M. (1987). Fine structure of the dorsal arm plate of Ophiocoma wendti: Evidence for a photoreceptor system (Echinodermata, Ophiuroidea). Zoomorphology, 107, 261-272.

Honey-Escandón, M., Solís-Marín, F.A. \& LaguardaFigueras, A. (2008). Equinodermos (Echinodermata) del Pacífico Mexicano. Revista de Biología Tropical, 56(3), 57-73.

Ives, J.E. (1889). Variation in Ophiura panamensis and Ophiura teres. Proceedings of the Academy of Natural Sciences of Philadelphia, 41, 76-77.

Kerstich, A. \& Bertsch, H. (2007). Sea of Cortez marine invertebrates. A guide for the pacific coast, México to Perú (2 ${ }^{\mathrm{a}}$ Ed.). EUA: Sea Challengers.

Koehler, R. (1907). Révision de la collection des ophiures du muséum d'histoire naturelle de Paris. Bulletin Scientifique de la France et de la Belgique, 41, 279-351.

Koehler, R. (1914). A contribution to the study of Ophiurans of the United States National Museum. Bulletin of the United States National Museum, 84, 1-173.

LeClair, E.E. (1996). Arm joint articulations in the Ophiuran brittlestars (Echinodermata: Ophiuroidea): a morphometric analysis of ontogenetic, serial, and interspecific variation. Journal of Zoology, 240, 245-275.

Ljungman, A.V. (1867). Om några nya arter af Ophiurider. Öfversigt af Kongliga Vetenskaps Akademiens Förhandlingar, 23(6), 163-166.

Ludwig, H. (1899). Jugendformen von Ophiuren. Sitzungsberichte der Preussischen Akademie der Wissenschaften, 1899, 210-235.

Luke, S.R. (1982). Catalog of benthic invertebrate collections, Echinodermata. Scripps Institution of Oceanography series, 82, 1-66. 
Lütken, C. F. (1856). Bidrag til kundskab om Slangestjernerne. II. Oversigt over de vestindiske Ophiurer. Videnskabelige Meddelelser fra Dansk Naturhistorisk Förening i Kjøbenhavn. 7, 1-19.

Lütken, C.F. (1859). Addimenta af historiam Ophiuridarum. Kongelige Danske Videnskabernes Selskab Biologiske Skrifter, 5(5), 177-271.

Lyman, T. (1860). Descriptions of new Ophiuridae, belonging to the Smithsonian Institution and to the Museum of Comparative Zoology at Cambridge. Proceedings of the Boston Society of Natural History, 7, 193-204.

Lyman, T. (1865). Ophiuridae and Astrophytidae. Memoires of the Museum of Comparative Zoology at Harvard, 1(1), 1-200.

Lyman, T. (1882). Report of the Ophiuroidea Dredged by H.M.S. Challenger, 1873-1876. Challenger Reports. Zoology, 5(14), 1-386.

Maluf, L.Y. (1988). Composition and distribution of the Central Eastern Pacific Echinoderms. Natural History Museum of Los Angeles County, Technical Reports, 2, 1-242.

Martínez-Morales, M. G. (2019). Ciclo reproductivo del ofiuroideo Ophiocomella alexandri (Lyman, 1860) y la influencia de la luna en la liberación de sus gametos en Bahía de La Paz BCS. (Tesis de maestría en Ciencias en Manejo de Recursos Marinos) Centro Interdisciplinario de Ciencias Marinas, Instituto Politécnico Nacional. México.

Martynov, A. (2010). Reassessment of the classification of the Ophiuroidea (Echinodermata), based on morphological characters. I. General character evaluation and delineation of the families Ophiomyxidae and Ophiacanthidae. Zootaxa. 2697: 1-154.

Müller, J. and Troschel, F. H. (1842). System der Asteriden.1. Asteriae. 2. Ophiuridae. Vieweg: Braunschweig. 134

Nielsen, E. (1932). Ophiurans from the Gulf of Panama, California, and the Strait of Georgia. Vidensk Medd Dan Naturhist Foren, 91, 241-346.

O'Hara, T.D., Byrne, M. \& Cisternas, P. (2004). The Ophiocoma erinaceus complex: another case of cryptic speciation in echinoderms. In T. Heinzeller \& J.H. Nebelsick (Eds.), Echinoderms, München: Proceedings of the 11th International Echinoderm Conference (pp. 537-542). Munich, Germany: Balkema, Leiden.

O’Hara, T.D., Hugall, A.F., Thuy, B., Stöhr, S. \& Martynov, A.V. (2017). Restructuring higher taxonomy using broad-scale phylogenomics: the living Ophiuroidea. Molecular Phylogenetic Evolution, 107, 415-430.

O’Hara, T.D., Stöhr, S., Hugall, A.F., Thuy, B., \& Martynov, A.V. (2018). Morphological diagnoses of higher taxa in Ophiuroidea (Echinodermata) in support of a new classification. European Journal of Taxonomy, $416,1-35$.

O'Hara, T.D., Hugall, A.F., Cisternas, P.A., Boissin, E., Bribiesca-Contreras, G., Sellanes, J., Paulay, G. \& Byrne, M. (2019). Phylogenomics, life history and morphological evolution of ophiocomid brittlestars. Molecular Phylogenetics and Evolution, 130, 67-80.

Pineda-Enríquez, T. (2011). Biodiversidad de equinodermos de la zona sur del Golfo de California de 0 a $300 m$ de profundidad (Tesis de licenciatura). Universidad Nacional Autónoma de México, México.

Solís-Marín, F.A., Reyes-Bonilla, H., Herrero-Pérezrul, M.D., Arizpe-Covarrubias, O. \& Laguarda-Figueras, A. (1997). Sistemática y distribución de los equinodermos de la bahía de La Paz. Ciencias Marinas, 23, 249-263.

Solís-Marín, F.A., Laguarda-Figueras, A., Durán-González, A., Ahearn, C.G. \& Torres-Vega, J. (2005). Equinodermos (Echinodermata) del Golfo de California, México. Revista de Biología Tropical, 53, 123-137.

Spalding M.D., Fox H.E., Allen G.R., Davidson N., Ferdaña Z.A., Finlayson M., Halpern B.S., Jorge M.A., Lombana A., Lourie S. A., Martin K. D., McManus E., Molnar J., Recchia C.A. \& Robertson J. (2007). Ecoregions of the World: A Bioregionalization of Coastal and Shelf Areas, BioScience, 57(7), 573-583.

Steinbeck, J., \& Ricketts, E.F. (1941). Sea of Cortez. A leisurely journal of travel and research. New York, USA: Viking Press.

Stöhr, S. (2005). Who's who among baby brittle stars (Echinodermata: Ophiuroidea). Zoological Journal of the Linnean Society, 143, 543-576.

Sumner-Rooney, L., Rahman, I.A., Sigwart, J.D. \& Ullrich-Lüter, E. (2018). Whole-body photoreceptor networks are independent of 'lenses' in brittle stars. Proceedings of the Royal Society Biological Science, 285(1871).

Sumner-Rooney, L., Kirwan, J.D., Lowe, E. \& UllrichLüter, E. (2020). Extraocular Vision in a Brittle Star Is Mediated by Chromatophore Movement in Response to Ambient Light. Current Biology, 30(2), 319-327.

Verrill, A.E. (1867). Notes on the Radiata in the Museum of Yale College, with descriptions of new genera and species, (No. 2). Notes on the Echinoderms of Panama and West Coast of America, with descriptions of new genera and species. Transactions of the Connecticut Academy of Arts and Sciences, 1, 251-322.

Ziesenhenne, F.C. (1937). Echinoderms from the West Coast of Lower California, the Gulf of California and Clarion Island. Zoologica, 22, 209-239. 